Recepción: 28 / 03 / 2018

Aceptación: 22 / 05 / 2018

Publicación: 05 / 06 / 2018

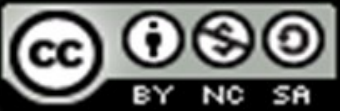

Ciencias económicas y empresariales Artículo de Investigación

\title{
El Coaching ejecutivo como herramienta de desarrollo del Talento Humano
}

\section{Executive Coaching as a tool for the development of Human Talent}

\section{Coaching Executivo como ferramenta para o desenvolvimento do Talento}

\section{Humano}

\author{
Silvia C. Villacis-Torres ${ }^{\mathrm{I}}$ \\ scvillacis@espe.edu.ec \\ Cecivel A. Velasco-Guachamin ${ }^{\text {II }}$ \\ cavelasco9@espe.edu.ec \\ Enma M. Torres-Espin ${ }^{\mathrm{III}}$ \\ emtorres1@espe.edu.ec
}

Correspondencia: scvillacis@espe.edu.ec

I Magister en Gerencia de Proyectos Educativos y Sociales, Especialista en Liderazgo y Gerencia, Diploma Superior en Diseño de Proyectos, Licenciada en Ciencias Administrativas, Administrador de Empresas, Ingeniera de Empresas, Docente de la ESPE, Latacunga, Ecuador.

II Ingeniera en Contabilidad y Auditoría CPA, Docente de la ESPE, Latacunga, Ecuador.

III Ingeniera Comercial, ESPE, Latacunga, Ecuador. 


\section{Resumen}

El propósito fundamental de este trabajo se centró en describir, primeramente, de qué trata el coaching ejecutivo, cuál es su concepto, quienes participan en ese proceso y cuáles son sus fases; para así entonces, explicar cómo es que ese proceso coadyuva al desarrollo del talento humano. Para ello, se desarrolló una investigación de tipo documental a nivel exploratorio-descriptivo. El Coaching Ejecutivo, es un acompañamiento no sólo para el directivo de la empresa sino para todo el personal involucrado, su proceso requiere de dos partes el Coach y el Coachee, y de una estructura para su óptimo desarrollo. Las fases básicas de dicho proceso se basan en: definir objetivos, involucrar a las partes, diseñar un plan de acción, establecer el feedback y feedforward de las partes y medir los resultados. En conclusión, el Coaching Ejecutivo es una de las herramientas que ha cobrado un mayor auge en la actualidad, no en vano, ya que ofrece una alta rentabilidad y una serie de cambios positivos y perdurables para mejorar las estrategias y/o los resultados organizacionales, este proceso resulta una buena opción como medio de desarrollar y explotar al máximo las capacidades del mayor recurso de la organización: el talento humano.

Palabras clave: Coaching; ejecutivo; desarrollo; talento; humano.

\section{Abstract}

The main purpose of this paper was to describe, first, what executive coaching is about, what its concept is, who participates in that process and what its phases are; to explain how this process contributes to the development of human talent. For this purpose, a documentary research was developed at an exploratory-descriptive level. Executive Coaching is an accompaniment not only for the manager of the company but for all personnel involved, its process requires two parts Coach and Coachee, and a structure for optimal development. The basic phases of this process are based on: defining objectives, involving the parties, designing a plan of action, establishing the feedback and feedforward of the parties and measuring the results. In conclusion, Executive Coaching is one of the tools that has gained a major boom today, not in vain, as it offers high profitability and a series of positive and lasting changes to improve strategies and / or organizational results, this process is a good option as a means to develop and exploit to the maximum the capabilities of the greatest resource of the organization: human talent.

Keywords: Coaching; executive; development; talent; human. 


\section{Resumo}

O propósito fundamental deste trabalho é centralizar-se, primeramente, de tratar o coaching ejecutivo, cuáli su concepto, quienes participan en ese proceso y cuáles son sus fases; for as entonces, explain cómo es that is proceso coadyuva al desarrollo del talento humano. Para ello, se desarrollou a uma investigação de tipo documental a nível exploratório-descritivo. The Coaching Executive, es un conpañamiento to the directio of the company sainte to the personal involucrado, and process rehabilitation of coach and the coach and the coachee, and de una estructura pour su óptimo desarrollo. As fases básicas do processo de decisão são: definição de objetivos, inclusão de um processo de decisão, estabelecimento de resultados e feedback das partes e resultados. En conclusión, el Coaching Ejecutivo es una de las herramientas that ha cobrado un mayor auge en la realidad, no en vano, ya that ofrece una alta rentabilidad y una série de cambios positivos y perdurables para mejorar las estrategias y / o los resultados organizationales, este é um processo de avaliação de como desenvolver e explicar as melhores capacidades de um recurso da organização: o talento humano.

Palavras chave: Coaching; ejecutivo; desarrollo; talento; humano.

\section{Introducción}

La competencia organizacional a nivel global, cada vez más se hace más intensa, las empresas que desean crecer o sobrevivir a esta sobre población empresarial deben emplear los recursos más modernos para asegurarse el éxito.

Uno de los principales recursos que posee una organización, en todos los niveles de su estructura, es el talento humano y quizás por mucho el más importante, pues de él dependen todas y cada una de las funciones de la empresa, desde el proceso productivo, pasando por el administrativo, hasta el mercadeo y posicionamiento del producto final, todos y cada uno de ellos, requieren del talento humano para su funcionamiento. Los casos de las industrias con un alto porcentaje de automatización de sus funciones no son la excepción, ya que, si bien es cierto que poseen menos personal que las empresas tradicionales, no es menos cierto que hasta la más alta tecnología requiere de personal que programe, opere y supervise las funciones automatizadas. Es por ello 
que el talento humano constituye el pilar fundamental de la organización, por tanto, resulta vital su efectiva gestión.

En este orden de ideas, partiendo de la prioritaria importancia que, tiene el talento humano surge la interrogante: ¿En qué lugar queda la organización?, y resulta interesante analizar la relación que tienen ambos desde el punto de vista de (Chiavenato, 2002), quien explica el mutualismo existente entre la organización y el talento humano de la siguiente manera:

Si la organización quiere alcanzar sus objetivos (crecimiento sostenido, rentabilidad, calidad en los productos y servicios, competitividad, entre otros.) de la mejor manera posible, debe saber canalizar los esfuerzos de las personas para que estas también alcancen sus objetivos individuales (mejores salarios, beneficios, estabilidad, satisfacción en el trabajo, oportunidad de crecimiento, entre otros.) y, de ese modo, se beneficien ambas partes. (p. 2)

Ahora bien, ese mutualismo que explica el mencionado autor constituye un matrimonio inseparable entre ambas partes cuya sana convivencia y permanencia efectiva debe ser garantizada por una buena gestión empresarial.

La gestión de talento humano, es definida por (Chiavenato, 2002), como:

Un área muy sensible a la mentalidad que predomina en las organizaciones. Es contingente y situacional, pues depende de aspectos como la cultura de cada organización, la estructura organizacional adoptada, las características del contexto ambiental, el negocio de la organización, la tecnología utilizada, los procesos internos y otra infinidad de variables importantes. Además, agrega que La gestión del talento humano en las organizaciones es la función que permite la colaboración eficaz de las personas (empleados, funcionarios, recursos humanos o cualquier denominación utilizada) para alcanzar los objetivos organizacionales e individuales. (p. 3,4)

La importancia de una efectiva gestión del talento humano ha llevado a las organizaciones a adoptar diversas herramientas para su efectivo desarrollo, es imperioso para la gestión del talento humano garantizar el mayor rendimiento de las personas para la empresa, así como la satisfacción para estas.

Existen muchas herramientas para gestionar talento humano, en el presente estudio se estudiará una de las más importantes y efectivas en la actualidad, se trata del Coaching Ejecutivo. 
El Coaching es un término muy escuchado en la actualidad y que aplica a diversos ámbitos en la vida cotidiana.

Según la International Coach Federation (2018), "el coaching profesional se fundamenta en una asociación con clientes en un proceso de acompañamiento reflexivo y creativo que les inspira a maximizar su potencial personal y profesional". (p. 1)

(Cattaneo, s.f.) se refiere a la historia del coaching mencionando que el Coaching data de hace muchos años, pero no es sino hasta la actualidad que se reconoce a nivel mundial su efectividad y se emplean sus técnicas, igualmente refiere que:

El coaching aparece en los años 80 y se consolida como una metodología con identidad propia. A rasgos generales se diferencian dos tipos de coaching: Coaching Personal o Coaching de Vida (Life Coaching) y Coaching Ejecutivo (Executive Coaching). Asimismo, la difusión del coaching se expande de formas diferentes según los diferentes contextos culturales, pero en general, en los últimos años su utilización, tanto en el entorno personal como sobre todo en el área empresarial, ha crecido exponencialmente. (p. 15)

\section{Metodología}

Se puede afirmar que, el presente trabajo básicamente se enmarca dentro de una investigación documental, debido a que la misma se ha basado en la consulta, tras la lectura, de una variedad de material bibliográfico relacionado al tema, el cual se halló tanto en textos escritos: libros, revistas especializadas, boletines, informes, tesis, artículos científicos y periodísticos, entre otros; como en material digital de la misma clase y categoría al que se accedió mediante una computadora con conexión a internet, en específico, a través de los motores de búsqueda Google Académico ${ }^{\circledR}$ y Google ${ }^{\circledR}$.

Según Tancara (1993), la investigación documental es "una serie de métodos y técnicas de búsqueda, procesamiento y almacenamiento de la información contenida en los documentos, en primera instancia, y la presentación sistemática, coherente y suficientemente argumentada de nueva información en un documento científico, en segunda instancia". (p.94)

Ahora, respecto a la investigación descriptiva, Niño (2011) asegura que: 
Su propósito es describir la realidad objeto de estudio, un aspecto de ella, sus partes, sus clases, sus categorías o las relaciones que se pueden establecer entre varios objetos, con el fin de esclarecer una verdad, corroborar un enunciado o comprobar una hipótesis. (p.34)

En el mismo orden de ideas, para Grajales (1996) los estudios descriptivos "buscan desarrollar una imagen o fiel representación (descripción) del fenómeno estudiado a partir de sus características. Miden variables o conceptos con el fin de especificar las propiedades importantes de comunidades, personas, grupos o fenómeno bajo análisis".

\section{Resultados}

\section{Coaching Ejecutivo}

Cattaneo (s.f.), refiere que es "un proceso de desarrollo para asistir a profesionales a alcanzar cambios positivos y duraderos en su liderazgo para mejorar la estrategia y/o los resultados organizacionales". (p. 6)

\section{Partes del Proceso (Coach-Coachee-Organización)}

Las partes del proceso del Coaching Ejecutivo están representadas por el Coach y el Coache, vamos a partir de su definición para analizar su relación. (Eleva Escuela de Coaching, s.f.) los define de la siguiente manera:

Coach es el profesional, con formación especializada en Coaching, que acompaña a las personas en su proceso de cambio. Mientras que el Coachee es la persona que hace y se responsabiliza del proceso de cambio para conseguir su objetivo. También se le puede llamar cliente, pero nunca paciente.

Muchos autores identifican una tercera parte en el proceso, la cual resulta innegable, ya que se trata de la Organización que para los efectos no necesita definición, sin embargo vamos a establecer que es quien contrará al Coach y quien tiene contratado al Coachee, la organización es el eje sobre el cual debe girar el proceso del Coaching Ejecutivo, ya que las mejoras que persigue para el Coachee repercuten en la satisfacción de las demandas de la Empresa. 


\section{Fases del Proceso}

\section{- Definir objetivos}

El primer paso para el proceso del Coaching Ejecutivo es la planificación, es fundamental puntualizar lo que la organización necesita, cuáles son sus expectativas para establecer metas claras.

Al inicio de un proceso de coaching, es cuándo se determina qué se quiere lograr. En este "horizonte" que se está creando, coach y coachee pueden determinar metas tangibles o materiales (conseguir mejorar las ventas un 20\%) o inmateriales (mejorar la relación con mi socio, etc...). Además, se establecen ciertos indicadores de cómo se medirá o se percibirá el éxito en la consecución de objetivos. (Piqueras, 2015)

\section{- Involucrar a los stakeholders}

Stakeholders, viene del inglés "partes involucradas", y este caso se refiere al talento humano que posee una organización, representado por todos y cada uno de sus niveles desde los gerenciales o líderes hasta el último eslabón de la cadena, considerado como un sistema cuyo buen funcionamiento es la base del éxito de la empresa.

El Coaching Ejecutivo, ciertamente está dirigido en primera instancia a los niveles ejecutivos de la organización, pero tomando en consideración lo anterior, este proceso no puede obviar el resto de los componentes de la empresa.

Tomando en consideración la importancia que tienen todas las partes en la organización, y la estrechez que todas y cada una deben tener en el proceso de Coaching Ejecutivo, (Gerens, 2017) explica seis formas en que las partes interesadas pueden ayudar en la resolución de un proyecto o conflicto:

1. Conocimiento: En las etapas iniciales del proyecto, usted puede involucrar a los stakeholders para ayudarle a comprender ciertos problemas. Los stakeholders pueden ayudarle a analizar detalladamente y comprender los conflictos y sus tonalidades. 
2. Comparación de opciones: Una vez que tenga algunas ideas respecto al proyecto, puede comprobarlas con los stakeholders para que le ayuden a considerar las ventajas y las desventajas de diferentes enfoques.

3. Priorización: Una vez que ha desarrollado cinco o seis áreas en las que podría enfocarse con éxito, puede involucrar a los grupos interesados para ayudarle a priorizar estas áreas.

4. Implementación: Los stakeholders pueden convertirse en socios claves al ayudarle en la implementación de un proyecto. Trabajar juntos es mejor que trabajar solo.

5. Animación: Los stakeholders pueden convertirse en triunfadores. Si los compromete desde el principio y los mantienes al corriente del progreso y la toma de decisiones, ellos estarán de acuerdo con lo que trate de lograr.

6. Evaluación: Las partes o grupos interesados pueden ser involucradas en la planificación de la evaluación y pueden informar al equipo sobre lo que está funcionando y qué no lo está haciendo desde su perspectiva.

\section{- Diseñar el plan de acción}

Un Plan de acción es un tipo de plan que prioriza las iniciativas más importantes para cumplir con ciertos objetivos y metas. De esta manera, un plan de acción se constituye como una especie de guía que brinda un marco o una estructura a la hora de llevar a cabo un proyecto. Es un elemento de suma importancia (Planificar), las acciones deben considerarse en una forma planificada, estructurada, secuencial y ordenada, deben plasmarse y enmarcarse en un Plan, que sea ejecutado con fechas claras de compromiso, responsabilidades y acciones específicas. (Franco, 2018)

\section{- Feedback y Feedforward de los stakeholders}

El Feedback es una palabra en inglés que traducida al español significa realimentación o retroalimentación.

Asimismo, (Anzorena, s.f.) en un artículo define a la fase del Feedback de los stakeholders como:

Una de las herramientas esenciales para el desempeño del liderazgo. El líder debe asumir la responsabilidad del desarrollo de los miembros de su equipo y facilitar los procesos de 
aprendizaje y cambio, y en tal sentido una de las formas de llevar a cabo estas responsabilidades es a través de la entrega de sus opiniones acerca de su desempeño. Igualmente, indica que esta retroalimentación para la acción que brinda el líder a los miembros de su equipo, no es algo que sólo deba realizase una vez al año en la "evaluación de desempeño", sino que es una herramienta de uso cotidiano que sirve para optimizar la perfomance de los integrantes del equipo, en virtud de que todo feedback tiene como finalidad reforzar, mejorar o corregir la forma de hacer las cosas o los comportamientos de las personas.

El Feedforward, por su parte, es una palabra compuesta en inglés que se traduce como "feed": alimentar, "forward": adelante y en su conjunto su traducción sería algo como prealimentación o alimentar hacia adelante o hacia el futuro.

(Goldsmith, 2007) autor del término FeedForward, explica al respecto:

El FeedForward es una herramienta evaluativa cuyo propósito es proveer a los individuos, equipos y a las organizaciones sugerencias que les ayuden a realizar un cambio positivo en sus comportamientos. En este sentido, la herramienta se basa en el principio de que se puede cambiar el futuro, más no el pasado. Esta herramienta ayuda a predecir y a enfocarse en un futuro positivo, no en un frustrado pasado.
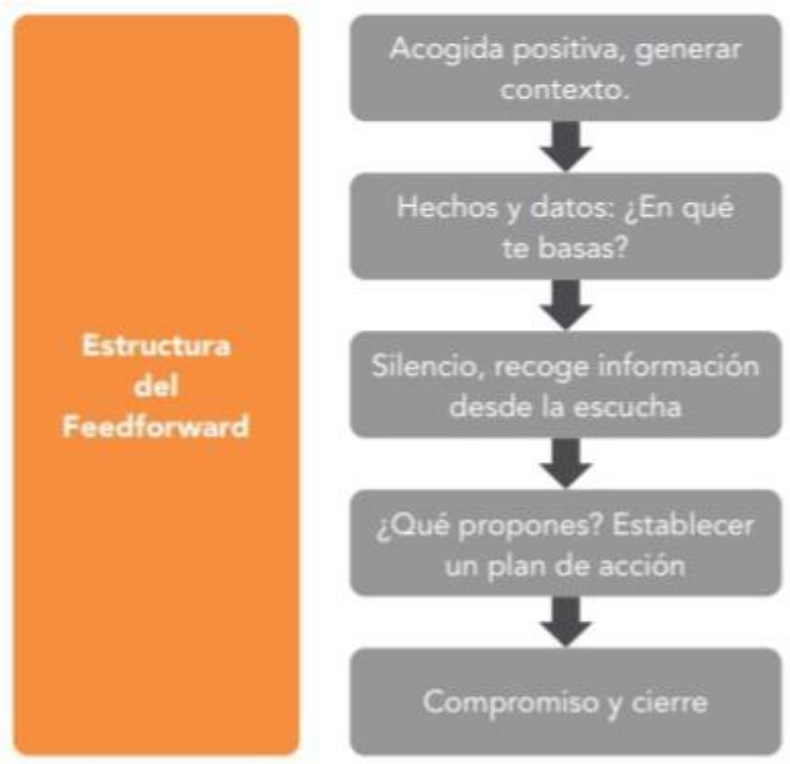

Imagen 1. Estructura del Feedworward.

Fuente: (Mascaraque, s.f.) 
(Mascaraque, s.f.) establece cinco pasos para realizar un Feedforward de calidad:

1. Acogida positiva: Cuando alguien se enfrenta a una conversación en la que le van a hablar de su desempeño, es muy probable que se sienta nervioso, es probable que este sentimiento entorpezca la comunicación. Es por eso la acogida debe ser un espacio corto donde se explique el contexto de la reunión y se cree un clima cálido.

2. Hechos y datos: ¿en qué te basas? Uno de los errores más comunes es dar un feedback poco preciso o basado, como ya hemos visto con anterioridad, en opiniones y observaciones apreciativas. En estos casos, se pueden dar y opiniones, pero desde lo concreto de los hechos.

3. Silencio, recoge información desde la escucha. Para conseguir que el feedforward, como el feedback, sea un diálogo en el que ambas partes crezcan y aprendan, es fundamental que la persona que ofrece el feedforward deje espacio para que el que lo reciba pueda también aportar su visión sobre lo que se está comentando.

4. ¿Qué propones? Establecer un plan de acción.

5. Compromiso y cierre: para cerrar la conversación, es fundamental concretar las responsabilidades asumidas por ambas partes. (p. 46-56)

Existe un problema fundamental con todos los tipos de feedback y es que se enfocan en el pasado, en lo que ya ocurrió, y no en la infinita variedad de oportunidades que pueden ocurrir en el futuro. El feedback puede ser limitado en lugar de ser expansivo y dinámico. Mientras que el FeedForward da sugerencias a las personas para el futuro, se ayuda a la persona tanto como se puede y esta a su vez escucha las sugerencias y aprende tanto como puede. (Goldsmith, 2007)

\section{- Medir resultados}

Existen diversas formas de medir los resultados del Coaching Ejecutivo, al respecto (Alto Nivel, 2010) explica:

La mejor forma de calcular el valor económico del cambio es considerar la mayor productividad, calidad, satisfacción del cliente y oportunidad de nuevos proyectos que genera el coaching. Asimismo, determinar la menor rotación de personal que provoca. Algunos elementos 
independientes que influyen en este proceso son las condiciones del mercado, acciones de la competencia o el cambio de tecnología.

\section{- Análisis del ROI (retorno de la inversión).}

Cuantifica la relación costo-beneficio del proceso y se enfoca netamente en los beneficios económicos. Establece la rentabilidad de la inversión comparada con otros proyectos u opciones de inversión disponibles. El análisis del ROI es una herramienta de suma utilidad para evaluar la conveniencia de un programa de mejora. Se requiere contemplar los costos asociados con la implementación y el mantenimiento del programa de mejora. Es indispensable establecer objetivos de negocio cuantificables que guíen y gobiernen el programa de mejora. Los beneficios económicos se determinan en base a estos objetivos de negocio. (Calvo \& Su, s.f.)

Por otra parte, (Calvo \& Su, s.f.) ejemplifican de manera práctica el cálculo del ROI de la siguiente manera:

El cálculo del ROI, se realiza dividiendo la estimación de los beneficios estimados entre los costos asociados con el programa. Si consideramos una inversión de 75 mil dólares, un beneficio esperado de 374 mil dólares al año tenemos:

$\mathrm{ROI}=374,400 / 75,000=4.99$

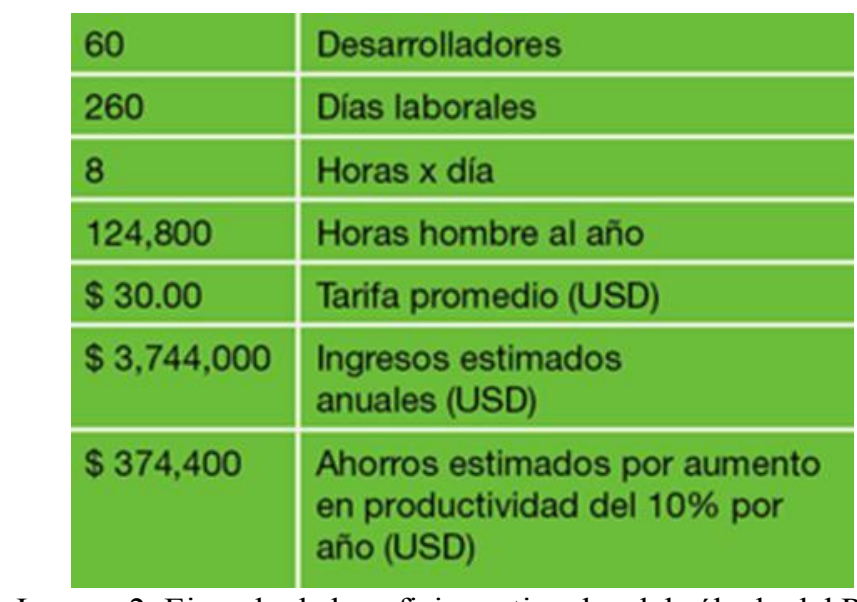

Imagen 2. Ejemplo de beneficios estimados del cálculo del ROI.

Fuente: (Calvo \& Su, s.f.)

Teniendo el ROI establecido resulta sencillo medir si las proyecciones del mismo fueron o no alcanzadas al final del proceso del Caoching Ejecutivo. 


\section{- $\quad$ El marco de referencia del bienestar y del compromiso (WBEF).}

Este método de medición, a diferencia del ROI que se enfoca únicamente en los beneficios económicos o financieros y que ofrece una visión limitada de los beneficios potenciales del coaching ejecutivo, evalúa un espectro mucho más amplio del alcance del proceso. Desarrollado por Grant y Spence (2010), cuenta con dos dimensiones: nivel de bien-estar (alto-bajo) y el nivel de compromiso con el trabajo (alto-bajo). Básicamente mide el compromiso con el trabajo (alta energía, fuerte participación y alto desempeño de las funciones laborales), disminución de la tensión y el estrés, aumento de la resiliencia y el bien-estar (auto aceptación, noción de propósito en la vida, relaciones positivas con los demás y autonomía), así como el logro de los objetivos planteados. Todas ellas variables claves dentro de las organizaciones exitosas e innovadoras. (Experience Designers, 2017)

\section{Beneficios del Coaching Ejecutivo}

Si en algo beneficia el coaching ejecutivo a las empresas es en proporcionarles mejores trabajadores que tienen capacidad desde las altas instancias de lograr después equipos de trabajo más sólidos. Con este coaching, los ejecutivos llegan a mejorar sus habilidades o aprender nuevas técnicas que hagan su día a día más sencillo. La eficacia crece de forma considerable en todos los sentidos y se aprende no solo a estar preparados para el éxito, sino también para saber cómo reaccionar ante posibles fracasos. Estos ejecutivos que atraviesan un proceso de coach ejecutivo con éxito, pueden ayudar al desarrollo de su empresa con la obtención de nuevos logros y mejorar al mismo tiempo su presencia en la misma. Con este tipo de coaching los ejecutivos dibujan un camino a seguir lleno de optimismo que luego se esfuerzan en lograr y a través el cual también interactúan con sus empleados y los demás miembros que pueden estar dentro de su entorno de trabajo. Los beneficios del coaching ejecutivo van tan lejos que pueden ser lo que haga que una empresa obtenga la plantilla que de verdad merece por el talento que suman todos sus trabajadores. (Mesa, s.f.)

\section{Conclusiones}

Partiendo de la premisa de que el talento humano es el mayor recurso de una organización resulta fundamental para la misma lograr que este recurso evolucione hasta alcanzar los límites máximos de sus capacidades, esto se traduce en el alcance de los objetivos empresariales. 
En torno al talento humano gira una serie de circunstancias que lo hacen cambiante en muchos sentidos, un ser humano tiene múltiples necesidades para alcanzar su nivel óptimo al ejecutar alguna función, esto no escapa al ámbito laboral, requiere desde un ambiente propicio para realizar el trabajo hasta una motivación que le resulte satisfactoria para el mejor cumplimiento de su labor (sueldos, beneficios, entre otros).

En este sentido, los líderes empresariales tienen el compromiso y la ardua tarea de gestionar el talento humano para lograr los mejores resultados, es allí donde cumple un rol importante el Coaching Ejecutivo como proceso de acompañamiento a la alta gerencia empresarial, involucrando a todo el personal de una organización como un todo. El Coaching orienta a los líderes de manera que estos presten especial atención a esas circunstancias cambiantes que giran en torno al personal de la empresa y detectar que requiere para un mejor funcionamiento. Este proceso representa una herramienta de desarrollo del talento humano con un auge considerable, que lo posiciona en la actualidad como una de las inversiones más usadas para aumentar la capacidad y el desarrollo del talento humano, ya que se enfoca en la obtención de los mejores resultados para la organización.

\section{Referencias bibliográficas}

Alto Nivel. (18 de noviembre de 2010). altonivel.com. Recuperado el 05 de septiembre de 2017, de https://www.altonivel.com.mx/liderazgo/management/7037-retorno-de-inversion-en-elcoaching/

Anzorena, O. (s.f.). DPO.Consulting. Recuperado el 05 de septiembre de 2017, de file://C:/Users/Luis/Desktop/Aymee/articulos\%20cient\%C3\%ADficos\%20Aymee/Administraci \%C3\%B3n\%20-\%20Talento\%20Humano/Oscar\%20Anzorena.html

Calvo, A., \& Su, A. (s.f.). Análisis del ROI: Una Herramienta para Justificar la Mejora de Procesos. (S. S. Guru, Ed.) SG Software Guru, 13. Recuperado el 05 de septiembre de 2017, de https://sg.com.mx/revista/13/analisis-del-roi-una-herramienta-para-justificar-la-mejora-procesos

Cattaneo, G. (s.f.). Coach Ejecutivo para Líderes. Colonia Granada. Recuperado el 05 de septiembre

de 2017, de 
http://vip.guidocattaneo.com/descargas/Coaching_Ejecutivo_Para_L\%C3\%ADderes__Guido_Cattaneo.pdf

Chiavenato, I. (2002). Gestión de Talento Humano (8va ed.). McGraw Hill. Recuperado el 05 de septiembre de 2017, de https://valerojulio.files.wordpress.com/2012/09/libro-gestion-del-talentohumano-chiavenato.pdf

Eleva Escuela de Coaching. (s.f.). Recuperado el 05 de septiembre de 2017, de https://www.elevacoaching.es/que-es-coaching-coach-coachee/

Experience Designers. (17 de octubre de 2017). experiencedesigners.pe. Recuperado el 04 de noviembre de 2017, de http://www.experiencedesigners.pe/2017/10/27/evaluar-exito-coachingejecutivo/

Gerens. (28 de diciembre de 2017). gerens.pe. Recuperado el 03 de enero de 2018, de https:/gerens.pe/blog/involucrar-a-los-stakeholders-proyecto/

Goldsmith, M. (2007). marshallgoldsmithfeedforward.com. Recuperado el 03 de enero de 2018, de http://www.marshallgoldsmithfeedforward.com/espanol/FeedForwardTool-espanol.htm

Grajales, T. (1996). Los tipos o clases de investigación. Obtenido de tgrajales.net: http://tgrajales.net/investipos.pdf

International Coach Federation. (2017). International Coach Federation ICF. Recuperado el 03 de enero de 2018, de https:/www.icf-es.com/mwsicf/sobreicf/definicion-coaching-icfespana?format $=$ pdf

Mascaraque, T. (s.f.). Del feedback al feddworward (1 ed.). Bookboon.com. Recuperado el 04 de septiembre de 2017, de https://bookboon.com/es/del-feedback-al-feedforward-ebook

Mesa, J. (s.f.). grupo-pya.com. Recuperado el 03 de enero de 2018, de https://blog.grupopya.com/desarrollo-de-talento-con-coaching-ejecutivo/

Niño, V. (2011). Metodología de la Investigación. Bogotá, Colombia: Ediciones de la U. Obtenido de http://roa.ult.edu.cu/bitstream/123456789/3243/1/METODOLOGIA\%20DE\%20LA\%20INVEST IGACION\%20DISENO\%20Y\%20EJECUCION.pdf 
Piqueras, C. (14 de septiembre de 2015). coachingvalencia.es. Recuperado el 04 de septiembre de 2017, de https://coachingvalencia.es/las-fases-de-un-proceso-de-coaching-ejecutivo/

Tancara, C. (1993). La Investigación Documental. Temas Sociales (17), 91-106. Obtenido de http://www.revistasbolivianas.org.bo/pdf/rts/n17/n17a08.pdf 